\title{
Termination of reperfusion arrhythmia by coronary artery occlusion
}

\author{
Ever D Grech, David R Ramsdale
}

\begin{abstract}
Reperfusion arrhythmias may be a cause of sudden death in acute myocardial infarction. A patient underwent successful coronary artery recanalisation by primary percutaneous transluminal coronary angioplasty (PTCA) for acute myocardial infarction. In this patient an idioventricular rhythm with a severe haemodynamic effect developed within one minute of deflation of the PTCA balloon. Sinus rhythm was immediately restored by reinflating the balloon and thus reinterrupting the supply of oxygenated blood. This suggests that reperfusion with consequent reoxygenation is a primary factor in arrhythmogenesis.
\end{abstract}

(Br Heart f 1994;72:94-95)

Reperfusion arrhythmias were first recorded in the dog heart by Tennant and Wiggers in 1935. ${ }^{1}$ Until the advent of thrombolytic treatment and interventional recanalisation for acute myocardial infarction, little attention had been paid to this phenomenon in humans. Observations of arrhythmias associated with reperfusion of the ischaemic myocardium in animal models have led to concern that similar events in humans might contribute to serious arrhythmia and sudden death. We report a patient who underwent successful coronary artery recanalisation by primary percutaneous transluminal coronary angioplasty (PTCA) for acute myocardial infarction, in whom idioventricular rhythm with severe haemodynamic effect was induced. Sinus rhythm could only be immediately restored on reinflating the PTCA balloon thus reinterrupting the supply of oxygenated blood. This suggests that reperfusion with consequent reoxygenation is a primary factor in arrhythmogenesis.

\section{Case report}

A 65 year old man presented with a three and a half hour history of severe cardiac chest pain and electrocardiographic features compatible with acute inferior myocardial infarction. $\mathrm{He}$ was normotensive and in sinus rhythm. There was no history of angina or myocardial infarction. The patient was transferred to the catheter laboratory for primary percutaneous transluminal coronary angioplasty (PTCA).

Left ventricular angiography showed posterobasal hypokinesia. Coronary arteriography showed an occluded proximal dominant right coronary artery, distal to the sinus node artery. The left coronary artery was normal. The occluded artery was successfully

Figure Simultaneous electrocardiogram (top) and aortic pressure wave changes (bottom) during coronary artery occlusion showing sinus rhythm and normotension (left), and reperfusion resulting in idioventricular rhythm and severe hypotension (right).

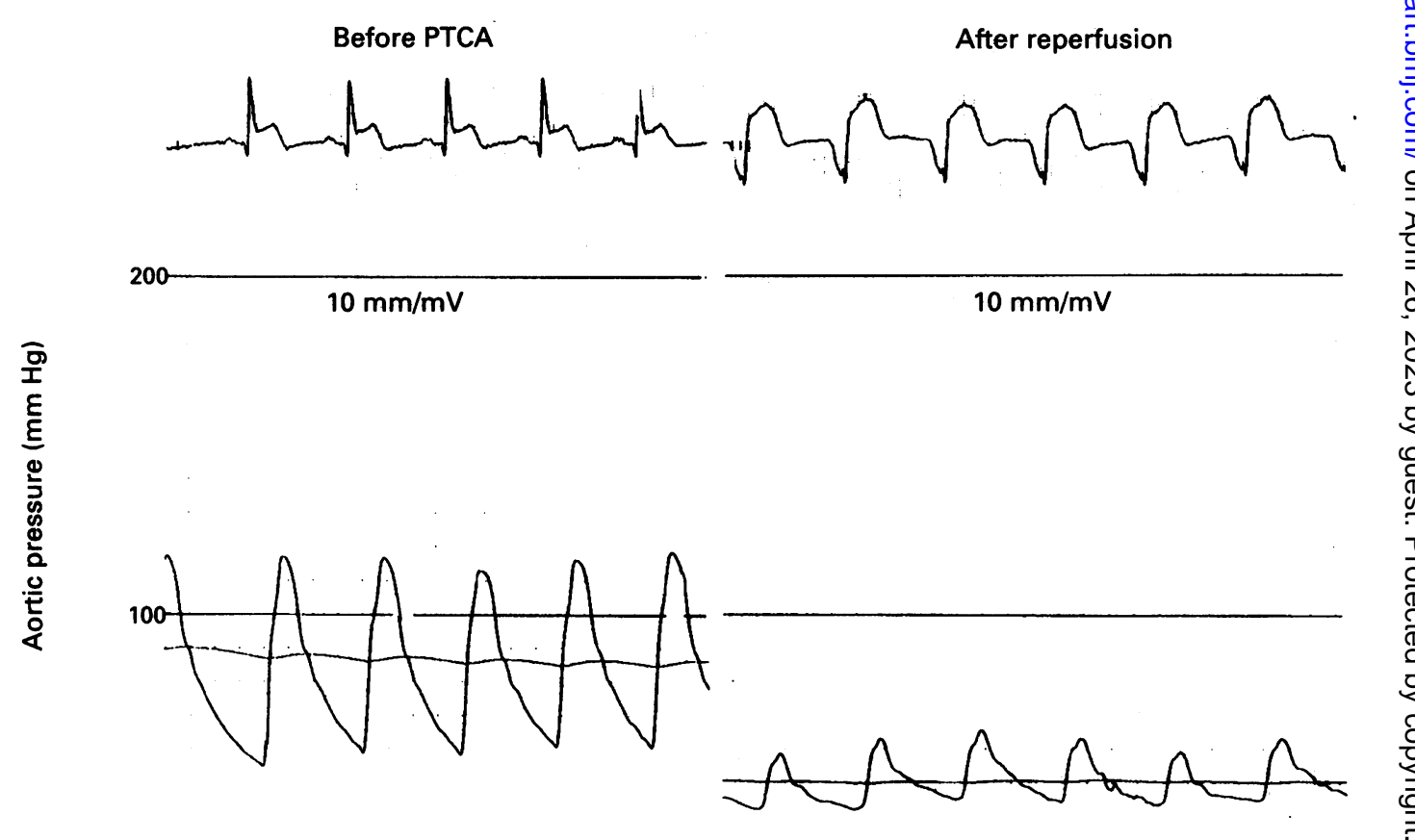


reopened by PTCA with a 0.014 inch high torque floppy guide wire and a $3.5 \mathrm{~mm}$ balloon dilatation catheter. Within one minute of balloon deflation, idioventricular rhythm developed (rate 93 beats $/ \mathrm{min}$ ); this was associated with a fall in blood pressure to $65 / 45$ $\mathrm{mm} \mathrm{Hg}$ (figure), dizziness, and nausea. Reinflation of the balloon catheter to improve the angiographic appearance of the underlying stenosis, produced a return to sinus rhythm and a blood pressure of $93 / 58 \mathrm{~mm} \mathrm{Hg}$ within 15 seconds. On balloon deflation idioventricular rhythm (rate 98 beats $/ \mathrm{min}$ ) again developed with associated hypotension $(75 / 55 \mathrm{~mm}$ $\mathrm{Hg}$ ). A third balloon inflation and deflation resulted in the same sequence of events. A $100 \mathrm{mg}$ intravenous bolus of lignocaine did not restore sinus rhythm, which spontaneously returned 20 minutes after the last balloon deflation. The blood pressure was stabilised on intravenous dobutamine and later adrenaline. Angiography 24 hours after recanalisation confirmed a widely patent right coronary artery without any further loss of posterobasal segmental contractility.

\section{Discussion}

During the acute phase of myocardial infarction, the mortality from arrhythmias is high. ${ }^{2}$ The restoration of coronary flow by spontaneous or pharmacological thrombolysis, or the mechanical disruption of the occlusive thrombus by coronary angioplasty, may paradoxically increase this risk.

Studies in humans have shown that arrhythmias, especially idioventricular rhythm, are common after reperfusion. ${ }^{3}$ The mechanisms responsible for ischaemic and reperfusion arrhythmias may be different. Whereas ischaemia may induce arrhythmias as a result of impaired energy supply and disturbances of ion homeostasis, the exact mechanisms of reperfusion arrhythmogenesis remain uncertain. Many hypotheses have been put forward including the accumulation of cellular ions and metabolites, the stimulation of adrenergic receptors, ${ }^{4}$ or the generation of cytotoxic oxygen derived free radicals causing disruption of cellular membrane function with resulting electrical instability. ${ }^{5}$

In humans, a further possibility is that reperfusion arrhythmias may be the result of microembolisation of the occlusive thrombus into the distal coronary bed. This may cause smaller areas of ischaemia and consequent ischaemic arrhythmias. In this patient, although mechanical disruption of the occluding thrombus by PTCA may have produced microemboli, the rapid starting and stopping of the idioventricular rhythm argues against this hypothesis. This report suggests that reoxygenation by reperfusion is an important factor in initiating arrhythmias. The exclusion of oxygenated blood (presumably containing electrophysiologically active compounds) by balloon reinflation resulted in a return to sinus rhythm on three occasions. There is strong evidence to show that reperfusion arrhythmias are mediated by oxygen derived free radicals. Studies with animal models have shown that these products are electrophysiologically active and that agents that remove free radicals can provide protection against reperfusion arrhythmias. ${ }^{6}$ In humans free radicals have been directly detected in coronary venous effluent blood in the immediate postreperfusion phase of acute myocardial infarction, ${ }^{7}$ although the effects of agents that remove free radicals on reperfusion arrhythmias have not been studied to date. As the mechanisms responsible for ischaemic and reperfusion arrhythmias are likely to be different, uniform antiarrhythmic treatment may not be appropriate for reperfusion arrhythmias.

1 Tennant R, Wiggers CJ. The effect of coronary occlusion on myocardial contraction. Am $\mathcal{F}$ Physiol 1935;112: on mi-61.

2 O'Doherty M, Taylor DI, Quinn E, Vincent R, Chamberlain DA. Five hundred patients with myocardial infarction monitored within one hour of symptoms. BMF 1983;286:1405-9.

3 Goldberg S. Greenspon AJ, Urban PL, et al. Reperfusion arrhythmia: a marker of restoration of antegrade flow during intracoronary thrombolysis for acute myocardial during intracoronary thrombolysis for

4 Kaplinski E, Ogawa S, Michelson EL, Dreifus LS Instantaneous and delayed ventricular arrhythmias after reperfusion of acutely ischemic myocardium: evidence for multiple mechanisms. Circulation 1981;63:333-40.

5 Pallandi RT, Perry MA, Campbell TJ. Proarrhythmic effects of an oxygen-derived free radical generating system on action potentials recorded from guinea pig ventricular myocardium: a possible cause of reperfusioninduced arrhythmias. Circ Res 1987;61:50-4.

6 Bernier M, Hearse DJ, Manning AS. Reperfusion-induced arrhythmias and oxygen-derived free radicals. Studies with "anti-free radical" interventions and a free radicalgenerating system in the isolated perfused rat heart. Circ Res 1986;58:331-40.

7 Grech ED, Dodd NJF, Bellamy CM, Perry RA, Morrison WL, Ramsdale DR. Free-radical generation during angioplasty reperfusion for acute myocardial infarction. Lancet 1993;341:990-1. 